\title{
MicroRNA-183 is involved in cell proliferation, survival and poor prognosis in pancreatic ductal adenocarcinoma by regulating Bmi-1
}

\author{
LIANG ZHOU $^{1 *}$, WEI-GUO ZHANG ${ }^{1 *}$, DE-SHENG WANG ${ }^{2 *}$, \\ KAI-SHAN TAO ${ }^{2}$, WEN-JIE SONG ${ }^{2}$ and KE-FENG DOU ${ }^{2}$ \\ ${ }^{1}$ Department of General Surgery, The 155 Central Hospital of PLA, Kaifeng, Henan 471000; \\ ${ }^{2}$ Department of Hepatobiliary Surgery, Xijing Hospital, The Fourth Military \\ Medical University, Xi'an, Shannxi 710032, P.R. China
}

Received May 5, 2014; Accepted July 3, 2014

DOI: $10.3892 /$ or.2014.3374

\begin{abstract}
As a highly aggressive malignant disease, the prognosis of patients with pancreatic ductal adenocarcinoma (PDAC) is poor. Yet, the mechanisms underlying the progression of PDAC remain unclear. MicroRNAs (miRNAs) may be involved in various human cancers as cancer suppressors or oncogenes. MicroRNA-183 (miR-183) was recently reported to be dysregulated in various types of cancer and to play an important role in the processes of cancer. However, the effects and potential mechanisms of action of miR-183 in PDAC have not been explored. In the present study, low expression of miR-183 was observed in PDAC tissues and cell lines. Low expression of miR-183 in PDAC was significantly associated with tumor grade, metastasis and TNM stage. Kaplan-Meier survival analysis demonstrated that patients harboring low expression of miR-183 had a significantly reduced overall survival than patients with a high level of miR-183 expression. The present study revealed that B-cell-specific Moloney murine leukemia virus insertion site 1 (Bmi-1) expression was inversely correlated with miR-183. Our findings also demonstrated that a low level of miR-183 expression effectively suppressed the growth of PDAC cells via regulation of Bmi-1. Following Bmi-1 silencing or upregulation of miR-183, the expression levels of cyclin D1, cyclin-dependent kinase (CDK)2 and CDK4 were decreased. It is reasonable to conclude that alteration of miR-183 expression may regulate the function of PDAC cells by the downregulation of Bmi-1 expression.
\end{abstract}

Correspondence to: Professor Ke-Feng Dou or Dr Wen-Jie Song, Department of Hepatobiliary Surgery, Xijing Hospital, The Fourth Military Medical University, Xi'an, Shannxi 710032, P.R. China

E-mail: kefengdou@126.com

E-mail:wjsong@fmmu.edu.cn

*Contributed equally

Key words: pancreatic ductal adenocarcinoma, microRNA-183, Bmi-1, proliferation, overall survival

\section{Introduction}

Pancreatic ductal adenocarcinoma (PDAC) is a highly aggressive malignant disease, and is ranked as the fourth leading cause of cancer-related mortality with a median survival of 6 months (1). Despite rapid advances in diagnostic and surgical procedures, PDAC remains one of the most difficult human malignancies to treat. Thus, identification of key molecules or pathways specifically expressed in PDAC that are essential for the growth and survival of cancer cells may provide novel therapeutic targets and ultimately lead to improved survival.

MicroRNAs (miRNAs) are a class of small, non-coding RNAs that play important roles in various biological processes (2). miRNAs have been discovered as naturally occurring non-coding RNAs, that control gene expression via specific sites at the 3'-UTR of target mRNAs, causing translational repression or degradation $(3,4)$. Recent evidence has shown that miRNA mutations or mis-expression is correlated with various human cancers and indicates that miRNAs can function as tumor suppressors or oncogenes. miR-183 is one member of the miR-182-183 miRNA cluster located in the 7q31-34 locus (5). Overexpression of miR-183 has been noted in human colorectal cancer, and the miR-183-96-182 cluster is frequently amplified in melanoma $(6,7)$. However, miR-183 was identified as a potential metastasis-inhibitor in lung cancer cells (8). These data suggest that the effect of miR-183 as an oncogene is cell-type dependent.

Although miR-183 and its target have been widely explored as cancer-related targets for tumors, there is no information available concerning their relevance in PDAC. The aim of the present study was to examine the role of miR-183 in PDAC and to investigate the potential mechanisms involved in miR-183 function in PDAC.

\section{Materials and methods}

Clinical samples and cell lines. PDAC tissues were obtained from the Department of Hepatobiliary Surgery, Xijing Hospital, The Fourth Military Medical University (Xi'an, China), between January 2003 and September 2008. Specimens were 
obtained from patients who had not received preoperative treatments such as chemotherapy. This study was approved by the Ethics Committee of The Fourth Military Medical University and conformed to the ethical guidelines of the 2004 Declaration of Helsinki. Written informed consent was obtained from each patient or from his/her legal guardians. Before the study was initiated, histopathological examinations were performed to confirm that there were enough cancer cells in the tumor samples and that no cancer cells had contaminated the non-cancerous tissues. Tissues were snap frozen in liquid nitrogen after surgical resection until use. The human pancreatic non-tumor cell line (HPDE6c7) and human PDAC cell lines (ASPC-1, SW1990, BXPC-3, CFPAC-1 and PANC-1) were cultivated in DMEM supplemented with $10 \%$ fetal calf serum (Sigma Chemical Co., St. Louis, MO, USA). Primary antibodies against Bim-1, cyclin D1, Cdk2, Cdk4 and GAPDH were purchased from Santa Cruz Biotechnology (Santa Cruz, CA, USA). All secondary antibodies were obtained from Pierce (Rockford, IL, USA). Bim-1 small interfering RNA (siRNA) and siRNA controls were obtained from Santa Cruz Biotechnology. Lipofectamine 2000 was purchased from Invitrogen (Carlsbad, CA, USA). All other chemicals and solutions were purchased from Sigma-Aldrich unless otherwise indicated.

Cell transfection. miR-183 mimics, inhibitors (miR-183-AS) and their respective negative controls (NC) were obtained from GenePharma Company (Shanghai, China). The day before transfection, cells were seeded in antibiotic-free medium. Transfections were carried out using Lipofectamine 2000 (Invitrogen, USA) in accordance with the manufacturer's instructions. To monitor transfection efficiency, fluorescein (FAM) siRNA (GenePharma) was used as control. Successfully transfected cells were observed with a fluorescence microscope.

siRNA cell transfection. According to the protocol supplied with the Lipofectamine 2000, the cells were transfected with either siRNA or control siRNA. siRNA-transfected cells were seeded into 6-well cell culture plates at a density of $1 \times 10^{5}$ cells/well. The cells were allowed to grow for an additional $24 \mathrm{~h}$ and were then harvested for further analysis.

Real-time PCR. Total RNA, including miRNAs, was isolated from prepared liver samples or cells with TRIzol reagent (Invitrogen) according to the manufacturer's instructions. Expression of hsa-miR-183 was analyzed with the miScript System (Qiagen, USA), which consists of the miScript Reverse Transcription kit, miScript Primer assays and miScript SYBRGreen PCR kit, according to the protocol provided by the company. Small nuclear RNA U6 was used for normalization. For the analysis of B-cell-specific Moloney murine leukemia virus insertion site 1 (Bmi-1) expression, cDNA was synthesized using Moloney murine leukaemia virus reverse transcriptase (Epicentre, Paris, France) as described by the manufacturer. The housekeeping gene, glyceraldehyde 3-phosphate dehydrogenase (GAPDH), was used for normalization. The forward primer for Bmi-1 was 5'-GCTTCAAGATGGC CGCTTG-3' and the reverse primer was 5'-TTCTCGTTGT TCGATGCATTTC-3'. The forward primer for GAPDH was 5'-GCACCGTCAAGGCTGAGAAC-3' and the reverse primer was 5'-TGGTGAAGACGCCAGTGGA-3'. Real-time PCR was run on the ABI Prism 7700 Sequence Detector (Applied Biosystems, USA). All of the reactions were run in triplicate. The ${ }^{\Delta \Delta} \mathrm{Ct}$ method was used for relative quantification of gene expression to determine miR-183 and Bmi-1 mRNA expression levels.

Protein extraction and western blotting. The cells were lysed in lysis buffer [50 mmol/l Tris (pH 7.5), $100 \mathrm{mmol} / \mathrm{l} \mathrm{NaCl}$, $1 \mathrm{mmol} / 1$ EDTA, 0.5\% NP40, 0.5\% Triton X-100, $2.5 \mathrm{mmol} / 1$ sodium orthovanadate, $10 \mu \mathrm{l} / \mathrm{ml}$ protease inhibitor cocktail and $1 \mathrm{mmol} / \mathrm{l} \mathrm{PMSF}$ ] by incubating for $20 \mathrm{~min}$ at $4^{\circ} \mathrm{C}$. The protein concentration was determined using the Bio-Rad assay system (Bio-Rad, Hercules, CA, USA). Total proteins were fractionated using SDS-PAGE and transferred onto nitrocellulose membranes. The membranes were blocked with $5 \%$ non-fat dried milk or bovine serum albumin in 1X TBS buffer containing $0.1 \%$ Tween-20 and then incubated with the appropriate primary antibodies. Horseradish peroxidase-conjugated anti-rabbit or anti-mouse IgG was used as the secondary antibody, and the protein bands were detected using the enhanced chemiluminescence detection system (Amersham Pharmacia Biotech). Quantification of the western blot analyses was performed using laser densitometry, and relative protein expression was then normalized to GAPDH levels.

Colony formation assay. Approximately $3 \times 10^{2}$ cells from each treated cell line were plated in 6-well dishes. After 2 weeks, cells were fixed with $20 \%$ methanol and stained with $1 \%$ crystal violet. Colonies consisting of $>50$ cells were counted per well, and each experiment was performed in triplicate.

Analysis of cell cycle. Both cell cycle distribution and spontaneous apoptosis events were detected using a FACSCalibur II sorter and Cell Quest FACS system (BD Biosciences, San Jose, CA, USA). To analyze cell cycle distribution, cells were synchronized using serum starvation for $24 \mathrm{~h}$ and stimulated with complete medium for $24 \mathrm{~h}$ before being harvested. Cells were fixed with $70 \%$ ethanol at $4^{\circ} \mathrm{C}$ overnight, washed twice with phosphate-buffered saline (PBS), and resuspended in staining solution (50 $\mathrm{lg} / \mathrm{ml}$ propidium iodide, $1 \mathrm{mg} / \mathrm{ml}$ RNase A, $0.1 \%$ Triton $\mathrm{X}-100$ in PBS) for $30 \mathrm{~min}$ at $37^{\circ} \mathrm{C}$ in the dark.

Cell growth assays. Cell growth was assessed using 3-(4,5-dimethylthiazol-2-y1)-2,5-diphenyltetrazolium bromide (MTT; Sigma) assays. Briefly, 2,000 cells/well were seeded into 96-well plates, and cell viability was assayed on days 1-4 following seeding. Absorption values were determined using an enzyme linked immunosorbent assay reader (Dasit, Milan, Italy) at $490 \mathrm{~nm}$.

Statistical analysis. Correlations between categorical variables were analyzed using Pearson's Chi-square, and two-tailed t-tests were used for continuous variables. Survival curves were plotted using the Kaplan-Meier method and were compared using the log-rank test. All statistical analyses were performed using the SPSS software package (SPSS, Chicago, IL, USA). A P-value $<0.05$ was considered to indicate a statistically significant result. 
Table I. Association of microRNA-183 expression and clinicopathologic factors of the PDAC patients.

\begin{tabular}{lccccc}
\hline & & \multicolumn{2}{c}{ miR-183 } & & \\
\cline { 3 - 4 } $\begin{array}{l}\text { Tumor } \\
\text { characteristics }\end{array}$ & $\mathrm{n}$ & Low & High & P-value & $\chi^{2}$ \\
\hline All cases & 91 & 47 & 44 & & \\
Gender & & & & & \\
$\quad$ Male & 56 & 29 & 27 & 0.974 & 0.001 \\
Female & 35 & 18 & 17 & & \\
Age (years) & & & & & \\
$\leq 50$ & 43 & 19 & 24 & 0.452 & 0.566 \\
$>50$ & 48 & 23 & 25 & & \\
Tumor grade & & & & & \\
(differentiation) & & & & & \\
$\quad$ Well & 27 & 22 & 5 & $<0.001$ & 13.682 \\
Moderately & 64 & 25 & 39 & & \\
or poorly & & & & & \\
Metastasis & & & & & \\
$\quad$ No & 57 & 22 & 35 & 0.001 & 10.407 \\
Yes & 34 & 25 & 9 & & \\
TNM stage & & & & & \\
I and II & 20 & 1 & 19 & $<0.001$ & 22.337 \\
III and IV & 71 & 46 & 25 & & \\
\hline
\end{tabular}

microRNA-183, miR-183; PDAC, pancreatic ductal adenocarcinoma.

\section{Results}

Low expression of miR-183 is associated with clinical progression of PDAC. To investigate the clinical role of miR-183 during pancreatic carcinogenesis, we analyzed its expression level in PDAC and compared it with clinicopathological features of the patients with PDAC. As shown in Table I, low expression of miR-183 in PDAC was significantly associated with tumor grade, metastasis and TNM stage $(\mathrm{P}<0.05)$. These results revealed a correlation between miR-183 expression and PDAC invasion and proliferation. However, there was no correlation between the expression of miR-183 and the other clinical features such as gender and age ( $\mathrm{P}>0.05$ for all comparisons). Furthermore, Kaplan-Meier survival analysis demonstrated that patients harboring low expression of miR-183 had a significantly reduced overall survival when compared with patients with a high level of miR-183 expression $(\mathrm{P}<0.001$, log-rank test; Fig. 1). These observations indicate that low expression of miR-183 is associated with PDAC clinical progression and may play a negative role in PDAC.

An inverse correlation exists between miR-183 and Bmi-1. To address the potential mechanism involved in the function of miR-183 in PDAC, we first examined the expression of miR-183 in the pancreatic non-tumor cell line, HPDE6c7, and in the PDAC cell lines, ASPC-1, SW1990, BXPC-3, CFPAC-1 and PANC-1 (Fig. 2). The results showed that, in the PDAC cells, the expression of miR-183 was lower than the level in

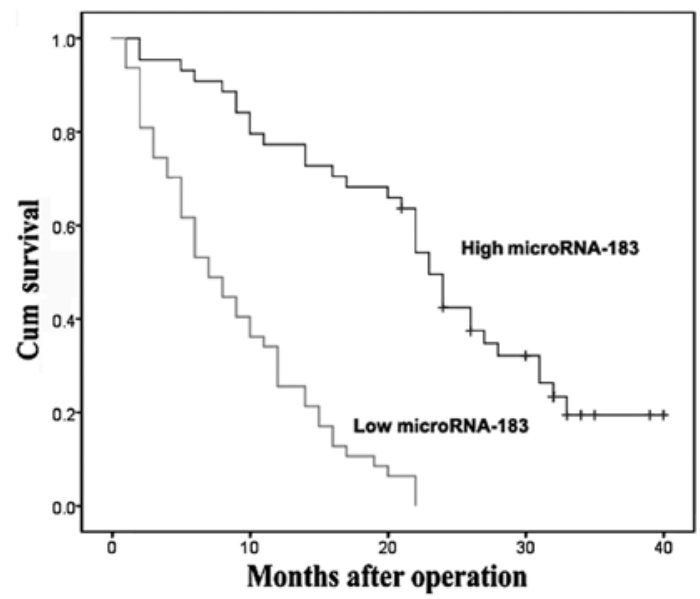

Figure 1. Kaplan-Meier statistical analysis of postoperative survival curves according to miR-183 expression. miR-183, microRNA-183.
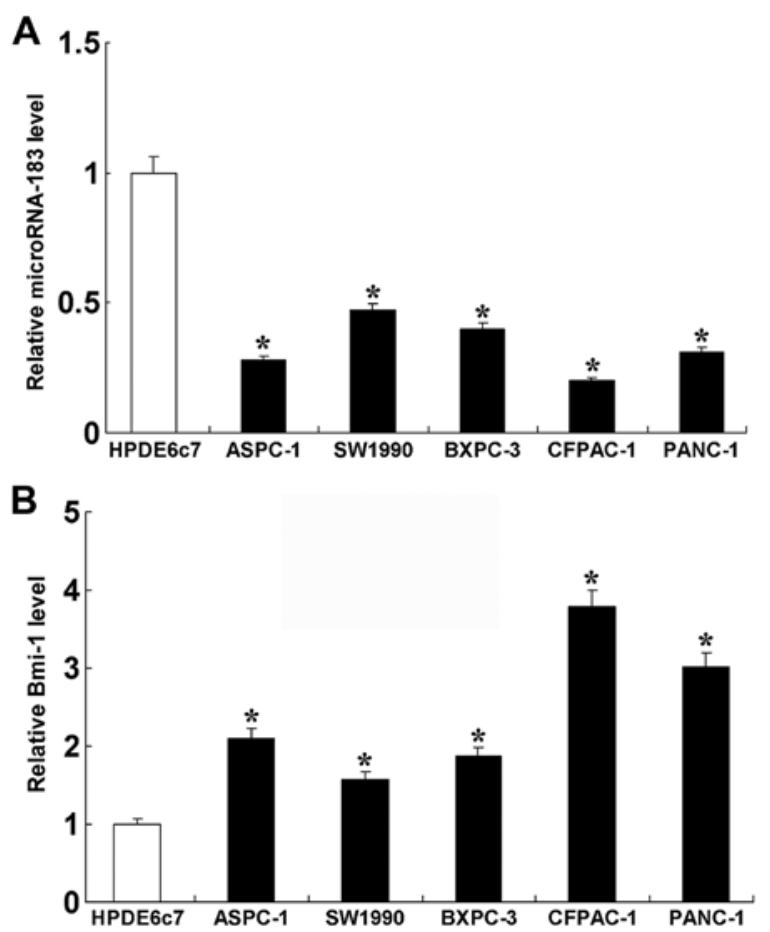

Figure 2. Expression of miR-183 and Bmi-1 in a pancreatic non-tumor cell line and PDAC cell lines. (A) RT-PCR analysis of the expression of miR-183 in the pancreatic non-tumor cell line, HPDE6c7, and in the PDAC cell lines, ASPC-1, SW1990, BXPC-3, CFPAC-1 and PANC-1. (B) RT-PCR analysis of the expression of Bmi-1 in a pancreatic non-tumor cell line and PDAC cell lines. The data represent means $\pm \mathrm{SD} ;{ }^{*} \mathrm{P}<0.05$ compared with HPDE6c7 cells. miR-183, microRNA-183; Bmi-1, B-cell-specific Moloney murine leukemia virus insertion site 1; PDAC, pancreatic ductal adenocarcinoma.

the non-tumor cells. We also examined the expression of Bmi-1 in the pancreatic non-tumor cell line and the PDAC cell lines. The results revealed that, in the PDAC cell lines, the expression of Bmi-1 was higher than that in the non-tumor cells. These results indicate that an inverse correlation exists between miR-183 and Bmi-1.

miR-183 regulates the protein expression of Bmi-1. To further examine the relationship between miR-183 and Bmi-1, we 

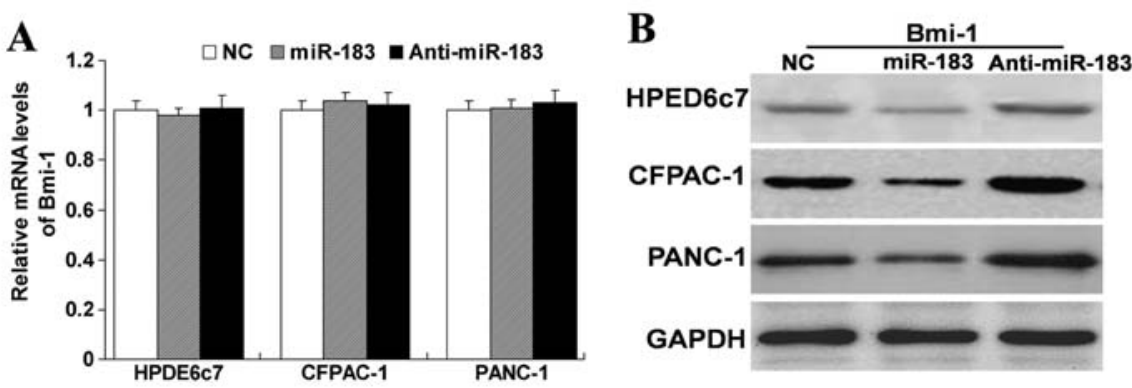

Figure 3. Bmi-1 is a target of miR-183. (A) HPDE6c7, CFPAC-1 and PANC-1 cells were transfected with miR-183 mimics or miR-183-AS or NC. The mRNA levels of Bmi-1 were analyzed by RT-PCR. (B) HPDE6c7, CFPAC-1 and PANC-1 cells were transfected with miR-183 mimics or miR-183-AS or NC. The protein levels of Bmi-1 were analyzed by western blotting. Bmi-1, B-cell-specific Moloney murine leukemia virus insertion site 1; miR-183, microRNA-183; NC, negative controls.
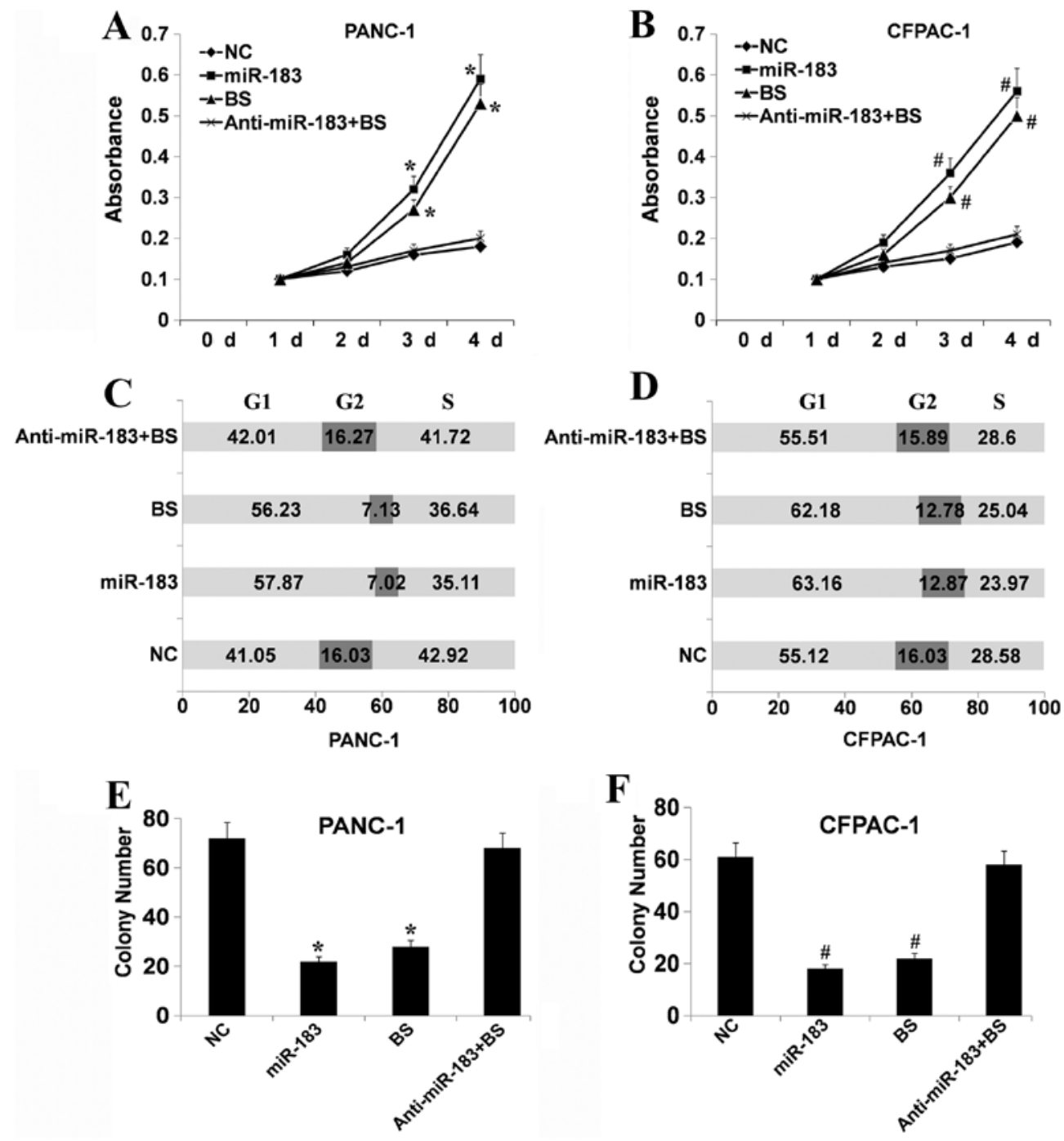

Figure 4. miR-183 regulates the function of cells via regulation of Bmi-1. (A and B) Cell viability of PANC-1 and CFPAC-1 cells was examined using MTT assays following treatment with miR-183 mimics or inhibitors and/or Bmi-1 siRNA (BS). (C and D) Cell cycle distribution was examined using flow cytometric analysis in the PANC-1 and CFPAC-1 cells following the different treatments. (E and F) Colony formation assays in the PANC-1 and CFPAC-1 following the different treatments. Data represent the means $\pm \mathrm{SD}$; ${ }^{\mathrm{P}}<0.05$ compared to negative control (NC)-transfected PANC-1 cells; ${ }^{*} \mathrm{P}<0.05$ compared to control negative control (NC)-transfected CFPAC-1 cells. miR-183, microRNA-183; Bmi-1, B-cell-specific Moloney murine leukemia virus insertion site 1.

treated the HPED6c7, CFPAC-1 and PANC-1 cells with miR-183 mimics or inhibitors. As shown in Fig. 3, we found that upregulation of miR-183 did not affect the mRNA expression of Bmi-1, but did decrease the protein expression of Bmi-1.
In contrast, downregulation of the expression of miR-183 did not affect the mRNA expression of Bmi-1, but did increase the protein expression of Bmi-1. These results indicate that miR-183 has a negative regulatory effect on Bmi-1. Yet, it was 

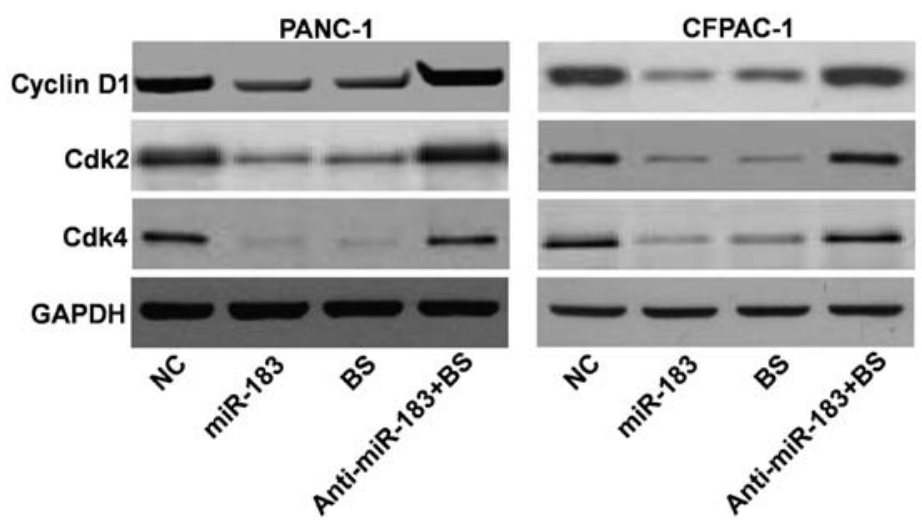

Figure 5. Changes in cell cycle-related protein expression in the PANC-1 and CFPAC-1 cells following treatment with miR-183 mimics or inhibitors and/or Bmi-1 siRNA (BS). Western blot analysis was used to detect the expression of several cell cycle-related regulatory factors in the PANC-1 and CFPAC-1 cells following the different treatments. GAPDH was used as the loading control. NC, negative controls.

unknown whether this negative regulation affects the function of cells. Thus, we carry out the subsequent experiment.

miR-183 regulates the function of cells via regulating Bmi-1. As known, Bmi-1 regulates the growth and proliferation of cells. To investigate whether miR-183 regulates the function of cells via regulation of Bmi-1, PANC-1 and CFPAC-1 cells, two cell lines with a low level of miR-183 and a high level of Bmi-1 expression, respectively, were treated with miR-183 mimics or inhibitors and/or Bmi-1 siRNA. Bmi-1 siRNA downregulated the expression of Bmi-1 (data not shown). We first examined the effect of the different treatments on cell viability using MTT assays. As shown in Fig. 4A and B, Bmi-1 depletion and upregulation of miR-183 significantly suppressed the growth of PANC-1 and CFPAC-1 cells compared with the control cells $(\mathrm{P}<0.05)$. In contrast, downregulation of miR-183 following downregulation of Bmi-1 had a slight influence on cell viability. This indicated that miR-183 may participate in cell growth via regulation of Bmi-1. Flow cytometric analysis showed that the cell cycle distribution of PANC-1 and CFPAC-1 cells was significantly affected following Bmi-1 depletion or upregulation of miR-183. As shown in Fig. $4 \mathrm{C}$ and D, there was a higher proportion of G0-G1 phase cells following Bmi-1 depletion or upregulation of miR-183 than in the control. A compensatory decrease in the $\mathrm{S}$ and G2/M phase proportions was also detected as compared with the control in the S and G2/M phases. Yet, downregulation of miR-183 following silencing of Bmi-1 had a slight influence. These results suggest that miR-183 can cause Bmi-1 silencing which inhibits the entry of cells into the $S$ phase therefore suppressing cell growth. Colony formation analysis showed that following Bmi-1 depletion or upregulation of miR-183, the PANC-1 and CFPAC-1 cells had a greatly reduced capacity to form colonies compared with the control cells (Fig. 4E and F). Downregulation of miR-183 following silencing of Bmi-1 had a slight influence. Collectively, these data showed that a low level of miR-183 expression effectively suppresses the growth of PDAC cells via the regulation of Bmi-1. Furthermore, western blotting was performed to explore the cell cycle regulatory role of miR-183. Following Bmi-1 silencing or upregulation of miR-183, the expression levels of cyclin D1, cyclin dependent kinase (CDK)2 and CDK4 were decreased (Fig. 5).

\section{Discussion}

The results of the present study revealed that miR-183 contributes to PDAC cell growth and proliferation via regulation of Bmi-1. These results elucidate the potential mechanisms and further confirm the importance and complexity of miR-183 in PDAC. To the best of our knowledge, this report is the first to describe the correlation of the miR-183-Bmi-1 pathway with PDAC cell growth and proliferation.

MicroRNAs (miRNAs) are small RNAs processed from endogenous transcripts that function to mediate post-transcriptional silencing of complementary target genes. miRNAs are a class of small non-coding regulatory RNA molecules, with a profound impact on various biological processes $(2,9,10)$. It has been reported that miRNAs are aberrantly expressed in most types of cancer where they are considered to play significant roles by regulating the expression of various tumor suppressors and oncogenes $(11,12)$. miR-183 is a member of an miRNA family (miR-183, miR-182 and miR-96) that is clustered within 2-4 kb at chromosome 7q32. miRNAs from this locus are dysregulated in a variety of tumors such as hepatic and colorectal, as well as in leukemia, lung and breast cancer (6,13-15). miR-183 family members have been shown to be upregulated in colorectal and hepatic tumors, as well as in leukemia and breast cancer (6,13-15). In contrast, miR-183 has been shown to be downregulated and inversely correlated with invasive and metastatic abilities in pulmonary giant cell (8) and breast cancer (16). These studies demonstrated that the expression profiling of miR-183 was tissue-specific and that it may have divergent functions depending on the tumor tissue or cell type.

Our results indicated that low levels of miR-183 expression in PDAC tissues are correlated with tumor grade, metastasis and TNM stage, each of which is an indicator of advanced tumor status. These results strongly suggest that miR-183 plays a key role in the progression of human PDAC. Kaplan-Meier analysis of the survival curves from patients in the present study showed a significantly worse overall survival rate for patients whose tumors had low miR-183 expression levels (log-rank test, $\mathrm{P}<0.001)$, indicating that low levels of miR-183 may serve as a marker of poor prognosis for patients with PDAC. To identify the potential role of miR-183 in PDAC, 
we compared the miR-183 expression level in a pancreatic non-tumor cell line and in PDAC cell lines, respectively. The results showed that, in the PDAC cell lines, the expression of miR-183 was lower than that in the non-tumor cell line. These results were similar to the results noted in the PDAC tissues. This indicates that miR-183 is a tumor suppressor in PDAC.

Since the potential mechanism of miR-183 as a tumor suppressor in PDAC was unknown, we explored the potential mechanism. We focused on B-cell-specific Moloney murine leukemia virus insertion site 1 (Bmi-1). Bmi-1 has been predicted to be a common target of miR-183 (17). Bmi-1, a member of the Polycomb family of proteins, which suppress the transcription of their target genes via an epigenetic mechanism (18-20), was originally identified as an oncogene cooperating with c-Myc in a murine lymphomagenesis model (21). Subsequent research identified the essential role of Bmi-1 in embryonic development and the maintenance of selfrenewal of both normal and malignant human mammary stem cells (22). Bmi-1 also regulates cellular processes including cell cycle progression, apoptosis and senescence as well as immortalization (23) and induces telomerase activity (24). In addition, there is accumulating evidence that Bmi-1 is overexpressed in a variety of human malignant neoplasms, such as melanoma (25) and HCC (26-28). Our previous study showed that Bmi-1 was overexpressed in PDAC cell lines and was associated with an unfavorable prognosis for patients with PDAC. When Bmi-1 was downregulated, cell growth was suppressed as a result of cell cycle arrest, and susceptibility to apoptosis was enhanced (29). Furthermore, Bmi-1 was found to be involved in tumor development and progression and is associated with a poor prognosis (30). For example, Bmi-1 expression is significantly correlated with nodal involvement, distant metastasis and clinical stage of colon and gastric cancers $(31,32)$. Overexpression of Bmi-1 has been associated with the invasiveness of nasopharyngeal carcinomas and was found to be a predictor of poor survival (33). Inhibition of Bmi-1 leads to decreased invasion of cervical cancer cells (34). Taken together, these data strongly indicate that Bmi-1 contributes to the behavior of cancer cells. Our study revealed that Bmi-1 expression was inversely correlated with miR-183. Our findings also demonstrated that Bmi-1 silencing and upregulation of miR-183 had the same effect on cellular processes including cell cycle progression and growth. In contrast, downregulation of miR-183 following silencing of Bmi-1 had only a slight influence on cell cycle progression and growth. These data revealed that low levels of miR-183 expression effectively suppressed the growth of PDAC cells via regulation of Bmi-1. Furthermore, western blotting was performed to explore the cell cycle regulatory role of miR-183. Following Bmi-1 silencing or upregulation of miR-183, the expression of cyclin D1, cyclin-dependent kinase (CDK)2 and CDK4 were decreased. It is reasonable to conclude that alteration of miR-183 expression may regulate the function of cells by targeting the downregulation of Bmi-1 expression.

In the present study, we demonstrated that upregulation of miR-183 has a similar role with inhibition of Bmi-1 in regulating PDAC cellular processes including cell cycle progression and growth. To the best of our knowledge, this is the first in vitro study to regulate the progression of PDAC, by regulation of miR-183 to target the expression of Bmi-1 in PDAC cells. The findings of this study revealed that by downregulating the Bmi-1 expression level, miR-183 plays a suppressive role in cellular processes including cell cycle progression and growth of PDAC. Our study may provide an important avenue for further analysis in vivo with the aim to develop a new potential diagnostic and therapeutic target for the screening and treatment of PDAC. Further studies are required to fully understand the regulatory mechanisms of miR-183 and Bmi-1 in PDAC in vitro and in vivo.

\section{Acknowledgements}

This study was supported by grants from the National Natural Science Foundation of China (grant no. 81101820).

\section{References}

1. Jemal A, Siegel R, Ward E, et al: Cancer statistics, 2008. CA Cancer J Clin 58: 71-96, 2008.

2. Bartel DP: MicroRNAs: genomics, biogenesis, mechanism, and function. Cell 116: 281-297, 2004.

3. Pillai RS, Bhattacharyya SN, Artus CG, et al: Inhibition of translational initiation by Let-7 microRNA in human cells. Science 309: 1573-1576, 2005.

4. Zamore PD and Haley B: Ribo-gnome: the big world of small RNAs. Science 309: 1519-1524, 2005.

5. Bastian BC, LeBoit PE, Hamm H, Bröcker EB and Pinkel D: Chromosomal gains and losses in primary cutaneous melanomas detected by comparative genomic hybridization. Cancer Res 58: 2170-2175, 1998.

6. Motoyama K, Inoue H, Takatsuno Y, et al: Over- and underexpressed microRNAs in human colorectal cancer. Int $\mathbf{J}$ Oncol 34: 1069-1075, 2009.

7. Lin WM, Baker AC, Beroukhim R, et al: Modeling genomic diversity and tumor dependency in malignant melanoma. Cancer Res 68: 664-673, 2008.

8. Wang G, Mao W and Zheng S: MicroRNA-183 regulates Ezrin expression in lung cancer cells. FEBS Lett 582: 3663-3668, 2008.

9. Ambros V and Lee RC: Identification of microRNAs and other tiny noncoding RNAs by cDNA cloning. Methods Mol Biol 265: 131-158, 2004.

10. Pillai RS, Bhattacharyya SN and Filipowicz W: Repression of protein synthesis by miRNAs: how many mechanisms? Trends Cell Biol 17: 118-126, 2007.

11. Caldas C and Brenton JD: Sizing up miRNAs as cancer genes. Nat Med 11: 712-714, 2005.

12. Kefas B, Godlewski J, Comeau L, et al: microRNA-7 inhibits the epidermal growth factor receptor and the Akt pathway and is down-regulated in glioblastoma. Cancer Res 68: 3566-3572, 2008.

13. Ladeiro Y, Couchy G, Balabaud C, et al: MicroRNA profiling in hepatocellular tumors is associated with clinical features and oncogene/tumor suppressor gene mutations. Hepatology 47: 1955-1963, 2008.

14. Agirre X, Jiménez-Velasco A, San José-Enériz E, et al: Downregulation of $h s a-m i R-10 a$ in chronic myeloid leukemia CD34 ${ }^{+}$ cells increases USF2-mediated cell growth. Mol Cancer Res 6: 1830-1840, 2008.

15. Mattie MD, Benz CC, Bowers J, et al: Optimized high-throughput microRNA expression profiling provides novel biomarker assessment of clinical prostate and breast cancer biopsies. Mol Cancer 5: 24, 2006.

16. Lowery AJ, Miller N, Dwyer RM and Kerin MJ: Dysregulated $m i R-183$ inhibits migration in breast cancer cells. BMC Cancer 10: 502, 2010.

17. Wellner U, Schubert J, Burk UC, et al: The EMT-activator ZEB1 promotes tumorigenicity by repressing stemness-inhibiting microRNAs. Nat Cell Biol 11: 1487-1495, 2009.

18. Jacobs JJ and van Lohuizen M: Polycomb repression: from cellular memory to cellular proliferation and cancer. Biochim Biophys Acta 1602: 151-161, 2002.

19. Kondo Y, Shen L, Cheng AS, et al: Gene silencing in cancer by histone H3 lysine 27 trimethylation independent of promoter DNA methylation. Nat Genet 40: 741-750, 2008. 
20. Raaphorst FM: Deregulated expression of Polycomb-group oncogenes in human malignant lymphomas and epithelial tumors. Hum Mol Genet 14 (Spec No 1): R93-R100, 2005.

21. van Lohuizen M, Verbeek S, Scheijen B, Wientjens E, van der Gulden $\mathrm{H}$ and Berns A: Identification of cooperating oncogenes in $\mathrm{E} \mu-m y c$ transgenic mice by provirus tagging. Cell 65: 737-752, 1991.

22. Liu S, Dontu G, Mantle ID, et al: Hedgehog signaling and Bmi-1 regulate self-renewal of normal and malignant human mammary stem cells. Cancer Res 66: 6063-6071, 2006.

23. Jacobs JJ, Kieboom K, Marino S, DePinho RA and van Lohuizen M: The oncogene and Polycomb-group gene bmi-1 regulates cell proliferation and senescence through the ink $4 a$ locus. Nature 397: 164-168, 1999.

24. Dimri GP, Martinez JL, Jacobs JJ, et al: The Bmi-1 oncogene induces telomerase activity and immortalizes human mammary epithelial cells. Cancer Res 62: 4736-4745, 2002.

25. Mihic-Probst D, Kuster A, Kilgus S, et al: Consistent expression of the stem cell renewal factor BMI-1 in primary and metastatic melanoma. Int J Cancer 121: 1764-1770, 2007.

26. Effendi K, Mori T, Komuta M, Masugi Y, Du W and Sakamoto M $\mathrm{Bmi}-1$ gene is upregulated in early-stage hepatocellular carcinoma and correlates with ATP-binding cassette transporter B1 expression. Cancer Sci 101: 666-672, 2010.

27. Sasaki M, Ikeda H, Itatsu K, et al: The overexpression of polycomb group proteins Bmi-1 and EZH2 is associated with the progression and aggressive biological behavior of hepatocellular carcinoma. Lab Invest 88: 873-882, 2008.
28. Wang H, Pan K, Zhang HK, et al: Increased polycomb-group oncogene Bmi-1 expression correlates with poor prognosis in hepatocellular carcinoma. J Cancer Res Clin Oncol 134: 535-541, 2008.

29. Song W, Tao K, Li H, et al: Bmi-1 is related to proliferation, survival and poor prognosis in pancreatic cancer. Cancer Sci 101: 1754-1760, 2010.

30. Sparmann A and van Lohuizen M: Polycomb silencers control cell fate, development and cancer. Nat Rev Cancer 6: 846-856, 2006.

31. Li DW, Tang HM, Fan JW, et al: Expression level of Bmi-1 oncoprotein is associated with progression and prognosis in colon cancer. J Cancer Res Clin Oncol 136: 997-1006, 2010.

32. Liu JH, Song LB, Zhang X, et al: Bmi-1 expression predicts prognosis for patients with gastric carcinoma. J Surg Oncol 97: 267-272, 2008.

33. Song LB, Zeng MS, Liao WT, et al: Bmi-1 is a novel molecular marker of nasopharyngeal carcinoma progression and immortalizes primary human nasopharyngeal epithelial cells. Cancer Res 66: 6225-6232, 2006.

34. Jiang Y, Su B, Meng X, et al: Effect of siRNA-mediated silencing of Bmi-1 gene expression on HeLa cells. Cancer Sci 101: 379-386, 2010. 